\title{
Communicating Correlated Sources over an Interference Channel
}

\author{
Arun Padakandla
}

\begin{abstract}
A new coding technique, based on fixed blocklength codes, is proposed for the problem of communicating a pair of correlated sources over a 2 -user interference channel. Its performance is analyzed to derive a new set of sufficient conditions. The latter is proven to be strictly less binding than the current known best, which is due to Liu and Chen [1]. Our findings are inspired by Dueck's example [2].
\end{abstract}

\section{INTRODUCTION}

Network information theory has provided us with elegant techniques to exploit correlation amongst distributed information sources. Such a correlation is handled at two levels. Probabilistic (soft) correlation is exploited via binning or transferring them via test channels [3]. When the sources possess common bits - Gács-Körner-Witsenhausen (GKW) common part -, conditional coding provides enhanced benefits. In this article, we propose a new coding technique to exploit the presence of near GKW parts, amongst distributed sources.

Our primary focus is the scenario depicted in Fig. 11. A pair $S_{1}, S_{2}$ of correlated sources have to be communicated over a 2 -user interference channel (IC). Receiver (Rx) $j$ wishes to reconstruct $S_{j}$ losslessly. We undertake a Shannontheoretic study and restrict attention to characterizing sufficient conditions under which $S_{j}$ can be reconstructed at $\operatorname{Rx} j$.

The current known best set of sufficient conditions (LC conditions) for this problem is due to Liu and Chen [1. Thm. 1] and are proven to be optimal for a class of deterministic ICs [1. Thm. 2]. In this article, we propose a new coding technique based on fixed block-length (B-L) codes and derive a new set of sufficient conditions. Through an example (Ex. 1), we prove (Lem. 2) the latter conditions are strictly less binding.

Presence of GKW part enables encoders co-ordinate their inputs, and thereby eliminate interference for the corresponding component of the channel input. Moreover, GKW part enables co-ordination even while enjoying separation. In other words, one can design a channel code corresponding to an optimizing input pmf, unconstrained by the source pmf. If $S_{1}, S_{2}$ do not possess a GKW part, a single-letter (S-L) technique is constrained by the S-L long Markov chain (LMC) $X_{1}-S_{1}-S_{2}-X_{2}$. The S-L LMC can, in general, severely constrain the set of achievable input pmfs (Ex. 1. Rem. 11). If $S_{1}, S_{2}$ possess a near $G K W$ part, i.e., $K_{j}=f_{j}\left(S_{j}\right): j \in[2]$ such that $\xi=P\left(K_{1} \neq K_{2}\right)$ is 'quite' small, (relatively) large sub-blocks of length $l$ could agree with high probability. Indeed, $\xi^{[l]}=P\left(K_{1}^{l} \neq K_{2}^{l}\right)=1-(1-\xi)^{l} \leq l \xi$ can be held small by appropriately choosing $l$. If the encoders employ conditional coding, i.e., identical source to channel mappings,

This work was supported by the Center for Science of Information (CSoI) an NSF Science and Technology Center, under grant agreement CCF-0939370.

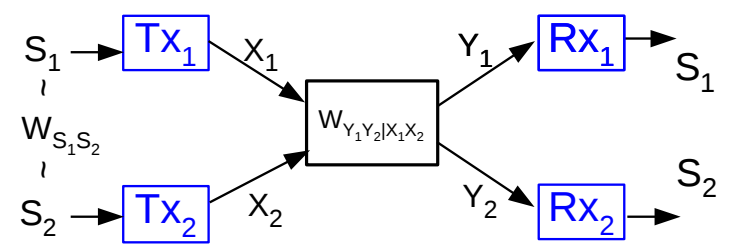

Fig. 1. Transmission of correlated sources over 2-IC.

restricted to sub-blocks of fixed length $l$, then the encoders can enjoy the benefits of separation and co-ordination on a good fraction (at least $\sim(1-l \xi)$ ) of these $l$-length sub-blocks. Indeed, we prove in Section III-A that the latter technique outperforms Liu and Chen's coding technique (LC technique). In Section IV] we build on this idea to propose a general coding technique for an arbitrary problem instance.

Joint source-channel coding over multi-user channels has received considerable attention with regard to characterizing fundamental limits $[4]-[6]$ and designing feasible strategies [7]. Fundamental performance limits for communicating Gaussian sources over Gaussian channels have been studied in [8], [9] [10] and the latter considers communication over on IC.

Our findings highlight the sub-optimality of (current known) S-L joint source-channel coding techniques (Rem. 2). Notwithstanding this, we derive a $S-L$ characterization (Rem. 4) of a new inner bound that strictly enlarges the current known best (LC bound). Indeed, the fixed B-L coding technique is an $l$-letter technique. An important second contribution is therefore, a framework - codes and tools (interleaving) for stitching together S-L techniques in a way that permits performance analysis of the resulting $l$-letter technique and characterization via S-L expressions. Stepping beyond performance characterization, our third contribution is a new coding technique for communicating correlated sources over an IC.

This is part of an evolving work [11]-[13] on joint sourcechannel coding, and is inspired by Dueck's novel example [2] and his very specific, yet ingenious, fixed B-L coding. Here, we restrict attention to separation based schemes 1 and focus on providing a clear step-by-step description of the ideas. Unifying fixed B-L coding and inducing source correlation onto channel inputs [14] involves additional challenges, and is dealt in a concurrent submission [12, Sec. V].

\section{Preliminaries : Notation, Problem statement}

We let an underline denote an appropriate aggregation of related objects. For example, $\underline{S}$ will be used to represent a pair $S_{1}, S_{2}$ of RVs. $\mathcal{S}$ will be used to denote either the

\footnotetext{
${ }^{1}$ As was done in $[11]$.
} 
pair $\mathcal{S}_{1}, \mathcal{S}_{2}$ or the Cartesian product $\mathcal{S}_{1} \times \mathcal{S}_{2}$, and will be clear from context. When $j \in\{1,2\}$, then $\dot{f}$ will denote the complement index, i.e., $\{j, j\}=\{1,2\}$. For $m \in \mathbb{N}$, $[m]:=\{1, \cdots, m\}$. For a pmf $p_{U}$ on $\mathcal{U}, b^{*} \in \mathcal{U}$ will denote a symbol with the least positive probability wrt $p_{U}{ }^{2}$ Boldfaces letters such as A denote matrices. For a $m \times l$ matrix $\mathbf{A}$, (i) $\mathbf{A}(t, i)$ denotes the entry in row $t$, column $i$, (ii) $\mathbf{A}(1: m, i)$ denotes the $i^{t h}$ column, $\mathbf{A}(t, 1: l)$ denotes $t^{t h}$ row. "with high probability", "single-letter", "long Markov chain", "block-length" are abbreviated whp, S-L, LMC, B-L respectively.

For a point-to-point channel (PTP) $\left(\mathcal{U}, \mathcal{Y}, \mathbb{W}_{Y \mid U}\right)$, let $E_{r}\left(R, p_{U}, \mathbb{W}_{Y \mid U}\right)$ denote the random coding exponent for constant composition codes of type $p_{U}$ and rate $R[15$, Thm 10.2]. Specifically, $E_{r}\left(R, p_{U}, \mathbb{W}_{Y \mid U}\right)$ is defined as

$$
\min _{V_{Y \mid U}}\left\{D\left(V_{Y \mid U}|| \mathbb{W}_{Y \mid U} \mid p_{U}\right)+\left|I\left(p_{U} ; V_{Y \mid U}\right)-R\right|^{+}\right\} \text {. }
$$

For RVs $A_{1}, A_{2}$, we let $\xi^{[l]}(\underline{A}):=P\left(A_{1}^{l} \neq A_{2}^{l}\right)$, and $\xi(\underline{A})$ : $=\xi^{[1]}(\underline{A})$. Throughout Sec. III $\xi^{[l]}=\xi^{[l]}(\underline{S})$ and $\xi=\xi(\underline{S})$. If $\underline{A}$ is IID, we not ${ }^{3} \xi^{[l]}=1-(1-\xi)^{l} \leq l \xi$. We let $\tau_{l, \delta}(K)=$ $2|\mathcal{K}| \exp \left\{-2 \delta^{2} p_{K}^{2}\left(a^{*}\right) l\right\}$ denote an upper bound on $P\left(K^{l} \notin\right.$ $\left.T_{\delta}^{l}(K)\right)$ where $T_{\delta}^{l}(K)$ denotes our typical set.

Consider a 2-user IC with input alphabets $\mathcal{X}_{1}, \mathcal{X}_{2}$, output alphabets $\mathcal{Y}_{1}, \mathcal{Y}_{2}$, and transition probabilities $\mathbb{W}_{Y_{1} Y_{2} \mid X_{1} X_{2}}$. Let $\underline{S}:=\left(S_{1}, S_{2}\right)$, taking values over $\underline{\mathcal{S}}:=\mathcal{S}_{1} \times \mathcal{S}_{2}$ with pmf $\mathbb{W}_{S_{1} S_{2}}$, denote a pair of information sources. For $j \in[2]$, encoder $j$ observes $S_{j}$, and decoder $j$ aims to reconstruct $S_{j}$ with arbitrarily small probability of error (Fig. 1). If this is possible, we say $\underline{S}$ is transmissible over $I C \mathbb{W}_{\underline{Y} \mid X}$. In this article, our objective is to characterize sufficient conditions under which $\left(\underline{\mathcal{S}}, \mathbb{W}_{\underline{S}}\right)$ is transmissible over IC $\mathbb{W}_{\underline{Y} \mid \underline{X}}$.

\section{FiXed B-L CODING OVER ISOLATED CHANNELS}

We consider a simple generalization (Ex. 1) of Dueck's example [2] and propose a coding technique that enables transmissibility of the sources over the corresponding IC. We also prove all current known joint source-channel coding techniques, and in particular LC, is incapable of the same. On the one hand, this proves strict sub-optimality of the latter ${ }^{4}$ and on the other hand, highlights the need for fixed BL coding.

Example 1: Source alphabets $\mathcal{S}_{1}=\mathcal{S}_{2}=\{0,1, \cdots, a-$ $1\}^{k}$. Let $\eta \geq 8$ be a positive even integer. The source PMF is

$\mathbb{W}_{S_{1} S_{2}}\left(c^{k}, d^{k}\right)= \begin{cases}\frac{k-1}{k} & \text { if } c^{k}=d^{k}=0^{k} \\ \frac{a^{\eta k}-1}{k a^{\eta k}\left(a^{k}-1\right)} & \text { if } c^{k}=d^{k}, c^{k} \neq 0^{k}, \\ \frac{1}{k a^{\eta k}\left(a^{k}-1\right)} & \text { if } c^{k}=0^{k}, d^{k} \neq 0^{k}, \text { and }\end{cases}$

0 otherwise. Note that in the above eqn. $c^{k}, d^{k} \in \mathcal{S}_{1}$ abbreviate the $k$ 'digits' $c_{1} c_{2} \cdots c_{k}$ and $d_{1} d_{2} \cdots d_{k}$ respectively. Fig. 2 depicts the source pmf with $\eta=6$.

\footnotetext{
${ }^{2}$ The underlying pmf $p_{U}$ will be clear from context.

${ }^{3}(1-x)^{l} \geq 1-x l$ for $x \in[0,1]$.

${ }^{4}$ Strict sub-optimality of LC technique can be inferred from [2]. To verify this, modify the MAC therein, to an IC with identical outputs, and use the arguments presented in proof of Lemma 1 Surprisingly, this has not been documented in [1].
}
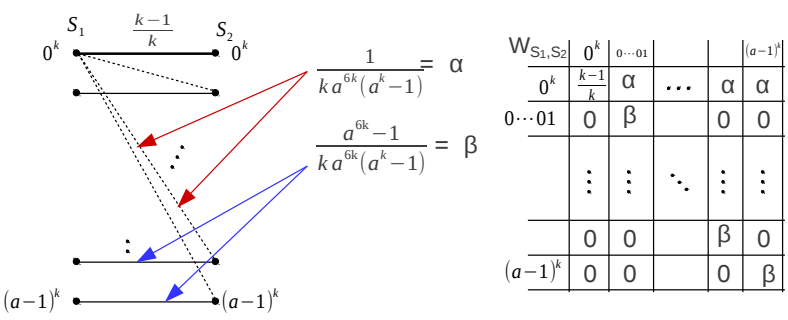

Fig. 2. On the left, the source pmf is depicted through a bipartite graph. Larger probabilities are depicted through edges with thicker lines. On the right, we depict the probability matrix.

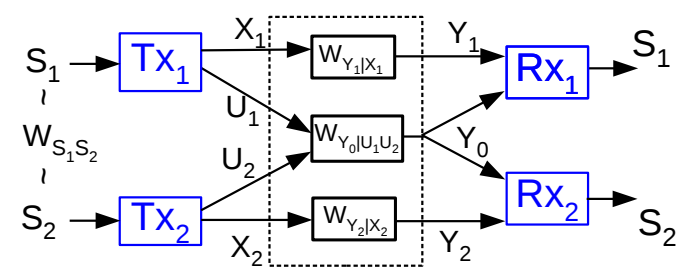

Fig. 3. Source channel setup of Example 1

The IC is depicted in Fig. 3 and described below. The input alphabets are $\mathcal{U} \times \mathcal{X}_{1}$ and $\mathcal{U} \times \mathcal{X}_{2}$. The output alphabets are $\mathcal{Y}_{0} \times$ $\mathcal{Y}_{1}$ and $\mathcal{Y}_{0} \times \mathcal{Y}_{2} . \mathcal{U}=\mathcal{Y}_{0}=\{0,1, \cdots, a-1\} .\left(U_{j}, X_{j}\right) \in \mathcal{U} \times$ $\mathcal{X}_{j}$ denotes encoder $j$ 's input and $\left(Y_{0}, Y_{j}\right) \in \mathcal{Y}_{0} \times \mathcal{Y}_{j}$ denotes symbols received by decoder $j$. The symbols $Y_{0}$ received at both decoders agree with probability $1 . \mathbb{W}_{Y_{0} Y_{1} Y_{2} \mid X_{1} U_{1} X_{2} U_{2}}=$ $\mathbb{W}_{Y_{1} \mid X_{1}} \mathbb{W}_{Y_{2} \mid X_{2}} \mathbb{W}_{Y_{0} \mid U_{1} U_{2}}$, where

$$
\mathbb{W}_{Y_{0} \mid U_{1} U_{2}}\left(y_{0} \mid u_{1}, u_{2}\right)= \begin{cases}1 & \text { if } y_{0}=u_{1}=u_{2} \\ 1 & \text { if } u_{1} \neq u_{2}, y_{0}=0, \text { and }\end{cases}
$$

0 otherwise. The capacities of PTP channels $\mathbb{W}_{Y_{j} \mid X_{j}}: j=1,2$ are $\mathcal{C}:=h_{b}\left(\frac{2}{k}\right)+\frac{2}{k} \log a$ and $\mathcal{C}+h_{b}\left(\frac{2}{k a^{\eta k}}\right)$ respectively.

We identify key aspects of Ex. 1] Let $a, k$ be chosen sufficiently/quiet large. While $\underline{S}$ does not possess a GKW part, $S_{1}$ and $S_{2}$ agree on most, but not all, realizations. Indeed, $\xi(\underline{S})=\frac{1}{k a^{\eta k}}$ is very small. We also have $H\left(S_{1}\right), H\left(S_{2}\right), H(\underline{S})$ are $\sim \log a$ and naturally $H\left(S_{j} \mid S_{j}\right): j \in[2]$ is very small. Each decoder benefits a lot by decoding either source, or any function thereof. Secondly, $\mathbb{W}_{\underline{S}}$ is 'very far' from the uniform pmf, and hence any S-L function $g_{j}\left(S_{j}\right)$ will remain 'considerably' non-uniform.

The IC supports a sum capacity of at most $\log a+2 \mathcal{C}+$ $h_{b}\left(\frac{2}{k a^{\eta k}}\right)$. Since $\sup _{p_{X_{1} X_{2}}} I(\underline{X} ; \underline{Y})=2 \mathcal{C}+h_{b}\left(\frac{2}{k a^{\eta k}}\right)$, the $\mathbb{W}_{Y_{0} \mid \underline{U}}$-channel must carry bulk of the information (for large $k)$. The latter channel carries very little information when $U_{1} \neq U_{2}$, and moreover, it is necessary that $U_{1}=U_{2}$ and $U_{1}=U_{2}$ be close to uniform, in order to communicate $\sim \log a$ bits over $\mathbb{W}_{Y_{0} \mid \underline{U}}$.

We first prove Ex. 1 does not satisfy LC conditions. The proof is based on the following argument. Suppose LC technique enables decoder $j$ reconstruct $S_{j}$ for $j \in$ [2], then both the decoders can reconstruct $S_{1}$ and $S_{2}$ if each of them is provided $Y_{1}$ and $Y_{2}$ (and $Y_{0}$ ). We then prove that this is not permissible, by following an argument similar to [2, Sec. III.c].

$$
{ }^{5} \text { In fact, }\left(1-\frac{1}{a^{\eta k}}\right) \log a-\frac{\log 2}{k} \leq \underset{H(\underline{S})}{H\left(S_{1}\right), H\left(S_{2}\right),} \leq \log a+h_{b}\left(\frac{1}{k}\right)+\frac{\log 2}{k}
$$


Lemma 1: Consider Ex. 1 with any $\eta \in \mathbb{N}$. There exists an $a_{*} \in \mathbb{N}, k_{*} \in \mathbb{N}$, such that for any $a \geq a_{*}$ and any $k \geq k_{*}$, the sources and the IC described in Ex. 1 do not satisfy LC conditions that are stated in [1, Thm. 1].

The proof is detailed in [13].

Proof: Since the sources do not have a GKW part, it suffices to prove that Ex. 11 does not satisfy conditions stated in [1, Corollary 1]. Let $\underline{S} Q \underline{W X U Y}$ be any collection of $\mathrm{RVs}$ whose pmf factorizes as $\mathbb{W}_{\underline{S}} p_{Q} p_{W_{1} \mid Q} p_{W_{2} \mid Q} p_{X_{1} U_{1} \mid S_{1} W_{1} Q} p_{X_{2} U_{2} \mid S_{2} W_{2} Q} \mathbb{W}_{\underline{Y} \mid X U}$. We prove

$$
\begin{array}{r}
H(\underline{S})>I\left(S_{1} X_{1} U_{1} ; Y_{1} Y_{0} \mid Q \underline{W}\right)+I\left(W_{1} S_{2} X_{2} U_{2} ; Y_{2} Y_{0} \mid Q\right) \\
-I\left(S_{1} ; S_{2}\right)
\end{array}
$$

and thereby contradicting [1, Eqn. (44), Corollary 2]. Towards that end, we first note

$$
\begin{aligned}
H(\underline{S}) & \geq H\left(S_{2}\right)=h_{b}\left(\frac{1}{k}\right)+\frac{1}{k} \log \left(a^{k}-1\right) \geq \frac{1}{k} \log \frac{k a^{k}}{2} \\
& \geq \log a+\frac{1}{k} \log \left(\frac{k}{2}\right) \geq \log a,
\end{aligned}
$$

whenever $a^{k} \geq 2$. Secondly, the RHS of (1) can be bounded above by

$$
\begin{aligned}
& I\left(S_{1} X_{1} U_{1} ; Y_{1} Y_{0} \mid Q \underline{W}\right)+I\left(W_{1} S_{2} X_{2} U_{2} ; Y_{2} Y_{0} \mid Q\right)-I\left(S_{1} ; S_{2}\right) \\
& \leq I\left(S_{1} X_{1} U_{1} ; Y_{1} Y_{0} \mid Q \underline{W}\right)+I\left(\underline{W} S_{2} X_{2} U_{2} ; \underline{Y} \mid Q\right)-I\left(S_{1} ; S_{2}\right) \\
& \leq \quad I(\underline{W S X U} ; \underline{Y} \mid Q)+I\left(S_{1} X_{1} U_{1} ; \underline{Y} \mid Q \underline{W}\right) \\
&-I\left(S_{1} X_{1} U_{1} ; \underline{Y} \mid Q \underline{W} S_{2} X_{2} U_{2}\right)-I\left(S_{1} ; S_{2}\right) \\
&= I(\underline{X U} ; \underline{Y} \mid Q)+I\left(S_{1} X_{1} U_{1} ; \underline{Y} \mid Q \underline{W}\right) \\
&-I\left(S_{1} X_{1} U_{1} ; \underline{Y} X_{2} U_{2} \mid Q \underline{W} S_{2}\right)-I\left(S_{1} ; S_{2}\right) \\
&= I(\underline{X U} ; \underline{Y} \mid Q)+I\left(S_{1} X_{1} U_{1} ; \underline{Y} \mid Q \underline{W}\right) \\
&-I\left(S_{1} X_{1} U_{1} ; \underline{Y} X_{2} U_{2} S_{2} \mid Q \underline{W}\right) \\
& \leq I(\underline{X U} ; \underline{Y} \mid Q)
\end{aligned}
$$

where (2) follows from Markov chains $Q \underline{S W}-\underline{X U}-\underline{Y}$ and $S_{1} X_{1} U_{1}-S_{2} Q \underline{W}-X_{2} U_{2}$ and (3) follows from the independence of $\underline{S}$ and $\underline{W} Q$.

We now argue the RHS above is strictly lesser than $\log a$. Our argument pretty much follows Dueck [2, Sec. III.c] verbatim and is provided here for the sake of completeness. Let $R=\mathbb{1}_{\left\{\left(S_{1}, S_{2}\right)=\left(0^{k}, 0^{k}\right)\right\}}$.

$$
\begin{array}{r}
I(\underline{X U} ; \underline{Y} \mid Q) \leq I(\underline{X U} R ; \underline{Y} \mid Q) \leq \log 2+I(\underline{X U} ; \underline{Y} \mid Q, R) \\
\leq \log 2+\frac{\log \left|\mathcal{Y}_{0} \times \mathcal{Y}_{1} \times \mathcal{Y}_{2}\right|}{k}+\left(1-\frac{1}{k}\right) I(\underline{X U} ; \underline{Y} \mid Q, R=1)
\end{array}
$$

We focus on the third term in the above sum. Conditioned on $R=1$, the sources are equal to $\left(0^{k}, 0^{k}\right)$. It can be verified that $X_{1} U_{1}-S_{1} Q-S_{2} Q-X_{2} U_{2}$. Given $Q=q, R=1,\left(X_{1}, U_{1}\right)$ is independent of $\left(X_{2}, U_{2}\right)$ and hence

$$
\begin{aligned}
I(\underline{X U} ; \underline{Y} \mid Q, R & =1) \leq \max _{p_{X_{1} U_{1} p_{X_{2} U_{2}}} I(\underline{X U} ; \underline{Y} \mid Q, R=1)} \leq 2 \mathcal{C}+h_{b}\left(\frac{2}{k a^{\eta k}}\right)+\max _{p_{U_{1}} p_{U_{2}} \mathbb{W}_{Y_{0} \mid \underline{U}}} H\left(Y_{0}\right)
\end{aligned}
$$

We now evaluate an upper bound on the maximum value of $H\left(Y_{0}\right)$ subject to $U_{1}, U_{2}$ being independent. We evaluate the following three possible cases.
Case 1a : For some $u \in \mathcal{U}, P\left(U_{1}=u\right) \geq \frac{1}{2}$ and $P\left(U_{2}=\right.$ $u) \geq \frac{1}{2}$. Then $P\left(Y_{0}=u\right) \geq \frac{1}{4}$ (independence of $\left.U_{1}, U_{2}\right)$ and hence $H\left(Y_{0}\right) \leq \log 2+\frac{3}{4} \log a$.

Case 1b : For some $u \in \mathcal{U}, P\left(U_{1}=u\right) \geq \frac{1}{2}$ and $P\left(U_{2}=\right.$ $u) \leq \frac{1}{2}$. Then $P\left(U_{2} \neq u\right) \geq \frac{1}{2}$ and hence $P\left(Y_{0}=0\right) \geq \frac{1}{4}$ and hence $H\left(Y_{0}\right) \leq \log 2+\frac{3}{4} \log a$.

Case 2a : For every $u \in \mathcal{U}, P\left(U_{1}=u\right) \leq \frac{1}{2}$. Then for any $u \in \mathcal{U}, P\left(U_{2} \neq U_{1}\right)=\sum_{u} \sum_{z \neq u} P\left(U_{2}=u\right) P\left(U_{1}=z\right) \geq$ $\frac{1}{2} \sum_{u} P\left(U_{2}=u\right)=\frac{1}{2}$, implying $P\left(Y_{0}=0\right) \geq \frac{1}{2}$ and hence $H\left(Y_{0}\right) \leq \log 2+\frac{3}{4} \log a$.

In all cases, we have $H\left(Y_{0}\right) \leq \log 2+\frac{3}{4} \log a$. Substituting through (3) and above, we conclude

$$
\begin{aligned}
I(\underline{X U} ; \underline{Y} \mid Q) \leq 2 \log 2+2 \mathcal{C}+h_{b}\left(\frac{2}{k a^{\eta k}}\right)+\frac{3}{4} \log a+\frac{\log |\mathcal{Y}|}{k} \\
<\log a
\end{aligned}
$$

for sufficiently large $k, a$.

Remark 1: Why is the LC technique incapable of communicating $\underline{S}$ ? Any valid pmf $p_{U_{1} U_{2}}$ induced by a S-L coding scheme is constrained to the LMC $U_{1}-S_{1}-S_{2}-U_{2}$. For $j \in$ [2], $p_{U_{j} \mid S_{j}}$ can equivalently be viewed as $U_{j}=g_{j}\left(S_{j}, W_{j}\right)$, for some function $g_{j}$ and RV $W_{j}$, that satisfy $W_{1} \Perp W_{2}$. Owing to the latter, $W_{1}$ and/or $W_{2}$ being non-trivial RVs, reduces $P\left(U_{1}=U_{2}\right)$. If we let, $W_{1}, W_{2}$ be deterministic, the only way to make $U_{j}$ uniform is to pool less likely symbols. However, the source is 'highly' non-uniform, and even by pooling all the less likely symbols, we can gather a probability, of at most, $\frac{1}{k}$. Consequently, any $p_{U_{1} U_{2}}$ induced via a S-L coding scheme is sufficiently far from any pmf that satisfies $U_{1}=U_{2}$ whp and $U_{1}=U_{2}$ close to uniform.

Remark 2: An $l$-letter (multi-letter with $l>1$ ) coding scheme is constrained by an $l$-letter LMC $U_{1}^{l}-S_{1}^{l}-S_{2}^{l}-U_{2}^{l}{ }^{6}$ Suppose we choose $l$ reasonably large such that 1$) \xi^{[l]}(\underline{S})$ is not high, and 2) $S_{j}^{l}$ is reasonably uniform on its typical set $T_{\delta}^{l}\left(S_{j}\right)$, and define $U_{j}: j \in[2]$ through identical functions $U_{j}^{l}=g\left(S_{j}^{l}\right): j \in[2]$, then one can easily visualize the existence of $g$ such that $p_{U_{1}^{l} U_{2}^{l}}$ satisfies the twin objectives of $U_{1}^{l}=U_{2}^{l}$ whp and $U_{1}^{l}=U_{2}^{l}$ is close to uniform. Our coding scheme, will in fact, identify such $g$ maps. This portrays the sub-optimality of S-L schemes for joint source-channel coding.

\section{A. Fixed Block-length Coding over a Noiseless Channel}

In order to input codewords on the $\mathbb{W}_{Y_{0} \mid \underline{U}}$-channel, that agree, we employ the same source code, same channel code and same mapping, each of fixed $B-L 7] l$, at both encoders. $l$ is chosen large enough such that the source can be reasonably efficiently compressed, and yet small enough, to ensure $\xi^{[l]}(\underline{S})$ is reasonably small. We refer to these $l$-length blocks as $s u b$ blocks. Since $l$ is fixed, there is a non-vanishing probability that these source sub-blocks will be decoded erroneously. An outer code, operating on an arbitrarily large number $m$ of these sub-blocks, will carry information to correct for these 'errors'. The outer code will operate over satellite channel $\mathbb{W}_{Y_{j} \mid X_{j}}$. We begin with a description of the fixed B-L codes.

\footnotetext{
${ }^{6}$ Constraint will not be via a S-L LMC.

${ }^{7}$ irrespective of the desired prob. of error
} 
We employ a simple fixed B-L (inner) code. Let $T_{\delta}^{l}\left(S_{1}\right)$ be the source code, and let $C_{U}=\mathcal{U}^{l}$ be the channel code. Let $l A=\left\lfloor\log a^{l}\right\rfloor$ bits, of the $\left\lceil\log \left|T_{\delta}^{l}\left(S_{1}\right)\right|\right\rceil$ bits output by the source code, be mapped to $C_{U}$. Both encoders use the same source code ${ }^{8}$ channel code and mapping.

Suppose we communicate an arbitrarily large number $m$ of these sub-blocks on $\mathbb{W}_{Y_{0} \mid \underline{U}}$ as above. Moreover, suppose encoder $j$ communicates the rest of the $l B=\left\lceil\log \left|T_{\delta}^{l}\left(S_{1}\right)\right|\right\rceil-$ $l A$ bits output by its source code to decoder $j$ on its satellite channel $\mathbb{W}_{Y_{j} \mid X_{j}} .^{9}$ How much more information needs to be communicated to decoder $j$, to enable it reconstruct $S_{j}^{l m}$ ? We do a simple analysis that suggests a natural coding technique.

View the $m$ sub-blocks of $S_{j}$ as the rows of the matrix $\mathbf{S}_{j}(1: m, 1: l) \in \mathcal{S}_{j}^{m \times l}$. Let $\hat{\mathbf{K}}_{j}(1: m, 1: l) \in \mathcal{S}_{1}^{m \times l}$ denote decoder $j$ 's reconstruction of $\mathbf{S}_{1}(1: m, 1: l)^{10}$. The $m$ sub-blocks

$$
\left\{\left(\mathbf{S}_{j}(t, 1: l), \hat{\mathbf{K}}_{j}(t, 1: l): j=1,2\right): t \in[m]\right\}
$$

are iiq ${ }^{11}$ with an $l$-length distribution $\mathbb{W}_{S_{1}^{l} S_{2}^{l}} p_{\hat{K}_{1}^{l} \hat{K}_{2}^{l} \mid S_{1}^{l} S_{2}^{12}}$ Since, in principle, we can operate by treating these $t$-length sub-blocks as a super-symbol, and employ standard binning technique over these $m$ super-symbols, decoder $j$ needs only $H\left(S_{j}^{l} \mid \hat{K}_{j}^{l}\right)$ bits per source sub-block. We have no characterization of $p_{\hat{K}_{1}^{l} \hat{K}_{2}^{l} \mid S_{1}^{l} S_{2}^{l}}$, and hence we derive an upper bound.

$$
\begin{gathered}
H\left(S_{j}^{l} \mid \hat{K}_{j}^{l}\right) \leq H\left(S_{j}^{l}, \mathbb{1}_{\left\{\hat{K}_{j}^{l} \neq S_{1}^{l}\right\}} \mid \hat{K}_{j}^{l}\right) \leq h_{b}\left(P\left(\hat{K}_{j}^{l} \neq S_{1}^{l}\right)\right)+ \\
+P\left(\hat{K}_{j}^{l} \neq S_{1}^{l}\right) \log \left|\mathcal{S}_{j}^{l}\right|+P\left(\hat{K}_{j}^{l}=S_{1}^{l}\right) H\left(S_{j}^{l} \mid S_{1}^{l}\right) . \\
\leq l \mathcal{L}_{l}^{S}\left(P\left(\hat{K}_{j}^{l} \neq S_{1}^{l}\right),\left|\mathcal{S}_{j}\right|\right)+l H\left(S_{j} \mid S_{1}\right), \text { where } \\
\mathcal{L}_{l}^{S}(\phi,|\mathcal{K}|):=\frac{1}{l} h_{b}(\phi)+\phi \log |\mathcal{K}|,
\end{gathered}
$$

represents the additional source coding rate needed to compensate for the errors in the fixed B-L decoding. It suffices to prove $\mathcal{L}_{l}^{S}\left(P\left(\hat{K}_{j}^{l} \neq S_{1}^{l}\right),\left|\mathcal{S}_{j}\right|\right)+B+H\left(S_{j} \mid S_{1}\right)<$ capacity of $\mathbb{W}_{Y_{j} \mid X_{j}}$. Since $\mathcal{L}_{l}^{S}(\phi,|\mathcal{K}|)$ is non-decreasing in $\phi$ if $\phi<\frac{1}{2}$, we bound $P\left(\hat{K}_{j}^{l} \neq S_{1}^{l}\right)$ by a quantity that is less than $\frac{1}{2}$. Towards that end, note that $\left\{S_{1}^{l} \neq \hat{K}_{j}^{l}\right\} \subseteq\left\{S_{1}^{l} \neq S_{2}^{l}\right\} \cup\left\{S_{1}^{l} \notin T_{\delta}^{l}\left(S_{1}\right)\right\}$. Indeed, $S_{1}^{l}=S_{2}^{l} \in T_{\delta}^{l}\left(S_{1}\right)$ implies both encoders input same $C_{U}$-codeword and agree on the $l B$ bits communicated to their respective decoders. Therefore $P\left(S_{1}^{l} \neq \hat{K}_{j}^{l}\right) \leq \phi$, where $\phi=\tau_{l, \delta}\left(S_{1}\right)+\xi(\underline{S})$,

$$
\tau_{l, \delta}\left(S_{1}\right) \leq 2 a^{k} \exp \left\{-\frac{\delta^{2} l}{2 k^{2} a^{2 k}}\right\} \text { and } \xi^{[l]}(\underline{S}) \leq \frac{l}{k a^{\eta k}} .
$$

Choose $l=k^{4} a^{\frac{\eta k}{2}}, \delta=\frac{1}{k}$, substitute in 77. Since $\eta \geq 6$, verify $\phi \leq 2 k^{3} a^{-\frac{\eta k}{2}}<\frac{1}{2}$ for sufficiently large $a, k$. Verify

\footnotetext{
${ }^{8}$ Encoder 2 also employs source code $T_{\delta}^{l}\left(S_{1}\right)$, (and not $T_{\delta}^{l}\left(S_{2}\right)$ ).

${ }^{9}$ Through our description, we assume communication over $\mathbb{W}_{Y_{j} \mid X_{j}}$ is noiseless. In the end, we prove that the rate we demand of $\mathbb{W}_{Y_{j} \mid X_{j}}$ is lesser than its capacity, justifying this assumption.

${ }^{10} 1$ ) Encoder $j$ could input any arbitrary $C_{U}$-codeword when its sub-block $S_{j}^{l} \notin T_{\delta}^{l}\left(S_{1}\right)$, and decoder $j$ could declare an arbitrary reconstruction when it observes $Y_{0}^{l}=0^{l}$. 2) $\hat{\mathbf{K}}_{2}(1: m, 1: l)$ is also viewed as reconstruction of $\mathbf{S}_{1}(1: m, 1: l)$.

${ }^{11}$ The $l$-length mappings from the source sub-blocks to the $l$-length channel codewords input on the $\mathbb{W}_{Y_{0} \mid \underline{U}}$ are identical across the sub-blocks and the source sub-blocks are mutually independent.

${ }^{12} p_{\hat{K}_{1}^{l} \hat{K}_{2}^{l} \mid S_{1}^{l} S_{2}^{l}}$ does not necessarily factor, owing to the $l$-length maps.
}

$$
\mathcal{L}_{l}^{S}\left(2 k^{3} a^{-\frac{\eta k}{2}},\left|\mathcal{S}_{j}\right|\right) \leq \frac{1}{4 k} \log a
$$

for sufficiently large $a, k$. Recall $l B=\left\lceil\log \left|T_{\delta}^{l}\left(S_{1}\right)\right|\right\rceil-l A$. Substituting $\delta=\frac{1}{k}$, verify 13

$$
B \leq(2 / l)+(1 / k) \log a+(1+(1 / k)) h_{b}(1 / k) .
$$

Since $h_{b}\left(\frac{2}{k}\right)-\left(1+\frac{1}{k}\right) h_{b}\left(\frac{1}{k}\right) \geq \frac{1}{2 k} \log \frac{k}{256}$ for large enough $k$, RHS of (8), 9) sum to at most $\frac{2}{l}+h_{b}\left(\frac{2}{k}\right)+\frac{5}{4 k} \log a$ for large enough $a, k$. Furthermore, $H\left(S_{2} \mid S_{1}\right) \leq h_{b}\left(\frac{1}{(k-1) a^{\eta k}}\right)+$ $\frac{2}{a^{\eta k}} \log a \leq h_{b}\left(\frac{2}{k a^{\eta k}}\right)+\frac{1}{4 k} \log a$ for sufficiently large $a, k$. It can now be easily verified that the satellite channels support these rates for large enough $a, k$.

A few details with regard to the above coding technique is worth mentioning. $p_{\hat{K}_{1}^{l} \hat{K}_{2}^{l} \mid S_{1}^{l} S_{2}^{l}}$ can in principle be computed, once the fixed block-length codes, encoding and decoding maps are chosen. $S_{j}^{l m}$ will be binned at rate $H\left(S_{j}^{l} \mid \hat{K}_{j}^{l}\right)$ and the decoder can employ a joint-typicality based decoder using the computed $p_{S_{j}^{l} \mid \hat{K}_{j}^{l}}$. We conclude the following.

Theorem 1: The LC conditions stated in [1. Thm. 1] are not necessary. Refer to Ex. 1 There exists $a^{*} \in \mathbb{N}$ and $k^{*} \in$ $\mathbb{N}$ such that for any $a \geq a^{*}$ and any $k \geq k^{*}, S_{1}, S_{2}$ and the IC $\mathbb{W}_{Y \mid X U}$ do not satisfy LC conditions, and yet, $\underline{S}$ is transmissible over IC $\mathbb{W}_{\underline{Y} \mid X U}$.

Remark 3: The above scheme crucially relies on the choice of $l$ - neither too big, nor too small. This is elegantly captured as follows. As $l$ increases, $\xi^{[l]}(\underline{S}) \rightarrow 1, \tau_{l, \delta}$ (and $\left.g_{\rho, l}\right)^{14} \rightarrow 0$. As $l$ decreases, $\xi^{[l]}(\underline{S}) \rightarrow \xi(\underline{S})$, and $\tau_{l, \delta}$ (and $\left.g_{\rho, l}\right) \rightarrow 1$. If $\phi \rightarrow 0.5, \mathcal{L}_{l}^{S}\left(\phi,\left|\mathcal{S}_{j}\right|\right) \rightarrow 0.5 \log \left|\mathcal{S}_{j}\right|=\frac{k}{2} \log a$.

\section{FIXED BL CODES OVER AN ARBITRARY IC}

Our analysis (Sec. III-A) focused on proving

$$
\mathcal{L}_{l}^{S}\left(\phi,\left|\mathcal{S}_{j}\right|\right)+B+H\left(S_{j} \mid S_{1}\right) \leq I\left(X_{j} ; Y_{j}\right)
$$

where $\phi<\frac{1}{2}$ was an upper bound on $P\left(\hat{K}_{j}^{l} \neq S_{1}^{l}\right)$. All our sufficient conditions will take this form. The lack of isolation between channels carrying fixed B-L and infinite B-L codes will throw primarily two challenges ${ }^{15}$ We present our generalization in three pedagogical steps.

In general, $P\left(S_{1}^{l} \neq \hat{K}_{j}^{l}\right) \leq \tau_{l, \delta}+\xi^{[l]}+g_{\rho, l}$, where the first two terms are as in (7), and $g_{\rho, l}$ is the probability that any of the decoders incorrectly decodes the $C_{U}$-codeword, conditioned on both encoders choosing the same $C_{U}$ codeword ${ }^{16}$ Our fixed B-L code $C_{U}$ will be a constant composition code, and in the statements of all theorems, $g_{\rho, l}$ is defined as

$$
g_{\rho, l}:=\sum_{j=1}^{2} \exp \left\{-l\left(E_{r}\left(A+\rho, p_{U}, p_{Y_{j} \mid U}\right)-\rho\right)\right\} .
$$

In all our theorems, $\mathcal{L}_{l}^{S}\left(\phi,\left|\mathcal{S}_{j}\right|\right)$ is defined as in $(6), g_{\rho, l}$ as above, $\phi=\tau_{l, \delta}\left(K_{1}\right)+\xi^{[l]}(\underline{K})+g_{\rho, l}$ will serve as an upper bound on $P\left(S_{1}^{l} \neq \hat{K}_{j}^{l}\right)$ that is less than $\frac{1}{2}$.

\footnotetext{
${ }^{13}$ Use $H\left(S_{1}\right) \leq \log a+h_{b}\left(\frac{1}{k}\right)$ and $\left|T_{\delta}\left(S_{1}\right)\right| \leq \exp \left\{l(1+\delta) H\left(S_{1}\right)\right\}$.

${ }^{14} g_{\rho, l}$ is defined in the sequel.

${ }^{15}$ And an additional loss in the channel rate, denoted $\mathcal{L}^{C}(\cdot, \cdot)$.

${ }^{16}$ For Ex $11 g_{\rho, l}=0$, and we ignored it. For general IC, $g_{\rho, l}$ is non-zero.
} 


\section{A. Designing independent streams ignoring self-interference}

The main challenges in generalizing pertain to 1) multiplexing a fixed B-L code with an infinite B-L code through a single channel input, and 2) the effect of erroneous conditional coding on the outer code. We adapt tools developed by Shirani and Pradhan [16], [17] in the context of distributed source coding. The following very simple generalization is chosen to illustrate our ideas. In particular, we live with self-interference between the two streams.

Theorem 2: $\left(\underline{\mathcal{S}}, \mathbb{W}_{\underline{S}}\right)$ is transmissible over IC $\left(\underline{\mathcal{X}}, \underline{\mathcal{Y}}, \mathbb{W}_{Y \mid X}\right)$ if there exists (i) a finite set $\mathcal{K}$, maps $f_{j}: \mathcal{S}_{j} \rightarrow \mathcal{K}$, with $K_{j}=f_{j}\left(S_{j}\right)$ for $j \in[2]$, (ii) $l \in \mathbb{N}, \delta>0$, (iii) finite set $\mathcal{U}, \mathcal{V}_{1}, \mathcal{V}_{2}$ and pmf $p_{U} p_{V_{1}} p_{V_{2}} p_{X_{1} \mid U V_{1}} p_{X_{2} \mid U V_{2}}$ defined on $\mathcal{U} \times \underline{\mathcal{V}} \times \underline{\mathcal{X}}$, where $p_{U}$ is a type of sequences 17 in $\mathcal{U}^{l}$, (iv) $A, B \geq 0, \rho \in(0, A)$ such that $\phi \in[0,0.5)$,

$$
A+B \geq(1+\delta) H\left(K_{1}\right), \text { and for } j \in[2],
$$

$B+H\left(S_{j} \mid K_{1}\right)+\mathcal{L}_{l}^{S}\left(\phi,\left|\mathcal{S}_{j}\right|\right)<I\left(V_{j} ; Y_{j}\right)-\mathcal{L}_{j}^{C}(\phi,|\mathcal{V}|)$,

where, $\phi:=g_{\rho, l}+\xi^{[l]}(\underline{K})+\tau_{l, \delta}\left(K_{1}\right), \mathcal{L}_{j}^{C}(\phi,|\mathcal{U}|)=h_{b}(\phi)+$ $\phi \log |\mathcal{U}|+|\mathcal{Y}||\mathcal{U}| \phi \log \frac{1}{\phi}$.

Remark 4: The characterization provided in Thm. 2 (and those in Thm. 3, 4) is via S-L PMFs and S-L expressions.

Remark 5: In the above, the fixed B-L code operates over $K_{j}^{l}$ instead of $S_{j}^{l} . \mathcal{L}_{j}^{C}(\phi,|\mathcal{U}|)$ quantifies the loss in rate of the outer code due to erroneous conditional coding. Note that, in Ex. 1, satellite channel remained unaffected when the encoders placed different $C_{U}$ codewords. The latter events imply, the $V_{j}-Y_{j}$ channel is not $p_{Y_{j} \mid V_{j}} \cdot \mathcal{L}_{j}^{C}(\phi,|\mathcal{U}|)$ is a bound on the difference in the mutual information between $p_{Y_{j} \mid V_{j}}$ and the actual channel. Note that $\mathcal{L}_{j}^{C}(\phi,|\mathcal{U}|) \rightarrow 0$ as $\phi \rightarrow 0$.

Proof: We elaborate on the new elements. The rest follows from standard arguments [13]. The source-coding module, and the mappings to the channel-coding module are identical to Section III-A. We describe the structure of $C_{U}$ and how it is multiplexed with the outer code built on $\mathcal{V}_{j}$. If we build a single code $C_{V_{j}}$ of B-L $l m$ and multiplex it with $m$ blocks of $C_{U}$, then $C_{V_{j}}$ does not experience an IID memoryless channel.

Let $\boldsymbol{U}_{j}(t, 1: l)$ denote encoder $j$ 's chosen codeword from $C_{U}$ corresponding to the $t^{\text {th }}$ sub-block of $\boldsymbol{K}_{j}(t, 1: l)$. We seek to identify sub-vectors of $\boldsymbol{U}_{j}$ that are IID, and whose pmf we know. We can then multiplex the outer code along these sub-vectors. Interleaving [17] enables us do this.

Suppose, for $t \in[m], \mathbf{A}(t, 1: l) \sim p_{A^{l}}$ and the $m$ vectors $\mathbf{A}(1,1: l), \cdots, \mathbf{A}(m, 1: l)$ are iid $p_{A^{l}}$. Let $\pi_{t}:[l] \rightarrow[l]: t \in$ $[m]$ be $m$ surjective maps, that are independent and uniformly chosen among the collection of surjective maps (permuters). Then, for each $i \in[l]$, the $m$-length vector

$\mathbf{A}\left(1, \pi_{1}(i)\right), \mathbf{A}\left(2, \pi_{2}(i)\right), \cdots, \mathbf{A}\left(m, \pi_{m}(i)\right) \sim \prod_{t=1}^{m}\left\{\frac{1}{l} \sum_{i=1}^{l} p_{A_{i}}\right\}$.

[12. Appendix A] contains a proof. The following notation will ease exposition. For $\mathbf{A} \in \mathcal{A}^{m \times l}$, and a collection $\pi_{t}$ : $[l] \rightarrow[l]: t \in[m]$ of surjective maps, we let $\mathbf{A}^{\pi} \in \mathcal{A}^{m \times l}$ be such that $\mathbf{A}^{\pi}(t, i):=\mathbf{A}\left(t, \pi_{t}(i)\right)$ for each $(t, i) \in[m] \times[l]$.

${ }^{17}$ Please refer to [15 Defn 2.1]
The above fact can be therefore be stated as $\mathbf{A}^{\pi}(1: m, i) \sim$ $\prod_{t=1}^{m}\left\{\frac{1}{l} \sum_{i=1}^{l} p_{A_{i}}\right\}$.

One can now easily prove that, if $C_{U}$ is a constant composition code of type $p_{U}$, and $m$ codewords are independently chosen ${ }^{18}$ from $C_{U}$ and placed as rows of $\boldsymbol{U}_{j}$, then for any $i \in[l]$, the interleaved vector $\mathbf{U}^{\pi}(1: m, i) \sim \prod_{t=1}^{m} p_{U}$. We now build $l$-codebooks (independently drawn), one for each of these interleaved vectors.

Following is our channel code structure. $C_{U}$ is constant composition code of type $p_{U}$ and B-L $l$. Encoder $j$ picks $l$ independent codes $C_{V_{j i}}: i \in[l]$, each iid $\sim \prod_{t=1}^{m} p_{V_{j}}(\cdot)$, each B-L $m . C_{V_{j i}}$ is multiplexed along with sub-vector $\boldsymbol{U}_{j}^{\pi}(1: m, i)$. Outer code message is split into $l$ equal parts $\left(M_{j 1}, \cdots, M_{j l}\right) . \boldsymbol{V}_{j} \in \mathcal{V}^{m \times l}$ is defined as $\boldsymbol{V}_{j}^{\pi}(1: m, i)=$ $C_{V_{j i}}\left(M_{j i}\right)$. For each $(t, i) \in[m] \times[l], \boldsymbol{X}_{j}(t, i)$ is chosen IID wrt $p_{X_{j} \mid U V_{j}}\left(\cdot \mid \boldsymbol{U}_{j}(t, i), \boldsymbol{V}_{j}(t, i)\right)$. Symbols in $\boldsymbol{X}_{j} \in \mathcal{X}_{j}^{m \times l}$ are input on the channel. It can be verified that (1) the codewords of $C_{U}$ and $C_{V_{j i}}$ pass through an IID memoryless channels whose transition probabilities are 'characterized' in the sequel.

Since each codebook $C_{V_{j i}}$ and each codeword is IID, $\boldsymbol{U}_{j}(t, 1: l) \rightarrow \boldsymbol{Y}_{j}(t, 1: l)$ is IID $p_{Y_{j} \mid U}$-channel. Interleaving ensures $\boldsymbol{V}_{j}^{\pi}(1: m, i) \rightarrow \boldsymbol{Y}_{j}^{\pi}(1: m, i)$ is IID. But, unless $\boldsymbol{U}_{1}=\boldsymbol{U}_{2}$, we are not guaranteed the latter channel is $\prod p_{Y_{j} \mid V_{j}}{ }^{19}$ In fact, we only know certain marginals of

$$
\boldsymbol{U}_{j}^{\pi}(1: m, i), \boldsymbol{V}_{j}^{\pi}(1: m, i), \boldsymbol{X}_{j}^{\pi}(1: m, i), \boldsymbol{Y}_{j}^{\pi}(1: m, i): j \in[2]
$$

Let $\left(\boldsymbol{V}_{j}^{\pi}(1: m, i), \boldsymbol{Y}_{j}^{\pi}(1: m, i)\right) \sim \prod p_{\hat{V}_{j} \hat{Y}_{j}}$, where $p_{\hat{V}_{j}}=p_{V_{j}}$. We wish to bound the difference $I\left(V_{j} ; Y_{j}\right)-$ $I\left(\hat{V}_{j} ; \hat{Y}_{j}\right)$ from above. Using the relations $p_{\hat{U}_{j}}=p_{U}$, $p_{\hat{V}_{j} \hat{Y}_{j} \mid \hat{U}_{1} \hat{U}_{2}}(v, y \mid u, u)=p_{V_{j} Y_{j} \mid U}(v, y \mid u)$, where $\prod p_{\hat{U}_{1} \hat{U}_{2}}$ is the pmf of the interleaved vector $\boldsymbol{U}_{1}^{\pi}(1: m, i) \boldsymbol{U}_{2}^{\pi}(1$ : $m, i), \quad \sum_{u_{1} \neq u_{2}} p_{\hat{U}_{1} \hat{U}_{2}} \leq \phi$, we can prove $\mid p_{V_{j} Y_{j}}(v, y)-$ $p_{\hat{V}_{j} \hat{Y}_{j}}(v, y) \mid \leq \phi$. These steps are analogous to those in [12 Appendix B]. Using [15, Proof of Lemma 2.7], we conclude $I\left(V_{j} ; Y_{j}\right)-I\left(\hat{V}_{j} ; \hat{Y}_{j}\right) \leq \mathcal{L}_{C}(\phi,|\mathcal{U}|)$. We refer to reader to [13] for the rest of the proof which is quite standard.

Lemma 2: The conditions stated in Thm. 2 are strictly weaker than those stated in [1, Thm. 1].

Proof: Ex. 1], with $a, k$ chosen sufficiently large, satisfies conditions stated in Thm 2. In particular, choose $\delta=\frac{1}{k}, \rho=$ $1, A=\left(1-\frac{1}{k^{3}}\right) \log a, B=H\left(S_{1}\right)-A, l=k^{4} a^{\frac{\eta k}{2}}, \mathcal{K}=\mathcal{S}_{j}$, $f_{j}=$ identity, $\mathcal{V}_{j}=\mathcal{X}_{j}, p_{U}$ uniform, $p_{V_{j}}$ capacity achieving. The result now follows from Lemma 1

\section{B. Additional information via Message-Splitting}

We now employ Han-Kobayashi technique to communicate the rest of the information (LHS of (10)). Towards that end, let $\mathscr{H} \mathscr{K}\left(p_{V_{1} W_{1} X_{1}} p_{V_{2} W_{2} X_{2}}\right)$ be the Han-Kobayashi inner bound defined in [1, Proposition 3].

Theorem 3: $\left(\underline{\mathcal{S}}, \mathbb{W}_{\underline{S}}\right)$ is transmissible over IC $\mathbb{W}_{\underline{Y} \mid \underline{X}}$ if there exists (i) a finite set $\mathcal{K}$, maps $f_{j}: \mathcal{S}_{j} \rightarrow \mathcal{K}$, with $\bar{K}_{j}=$ $f_{j}\left(S_{j}\right)$ for $j \in[2]$, (ii) $l \in \mathbb{N}, \delta>0$, (iii) finite set $\mathcal{U}, \mathcal{V}_{j}, \mathcal{W}_{j}$ : $j \in[2]$ and pmf $p_{U} p_{V_{1} W_{1}} p_{V_{2} W_{2}} p_{X_{1} \mid U V_{1} W_{1}} p_{X_{2} \mid U V_{2} W_{2}}$ defined

\footnotetext{
${ }^{18}$ Not necessarily uniformly. In fact the index output from the source code, owing to the fixed block-length $l$ is not necessarily uniform

${ }^{19} \mathrm{We}$ are unaware of the transition probabilities of this IID PTP.
} 
on $\mathcal{U} \times \underline{\mathcal{V}} \times \underline{W} \times \underline{\mathcal{X}}$, where $p_{U}$ is a type of sequences in $\mathcal{U}^{l}$, (iv) $A, B \geq 0, \rho \in(0, A)$ such that $\phi \in[0,0.5)$,

$$
A+B \geq(1+\delta) H\left(K_{1}\right), \text { and for } j \in[2],
$$

$\left(\begin{array}{c}B+\mathcal{L}^{C}(\phi,|\underline{\mathcal{U} \mathcal{W}}|) \\ H\left(S_{j} \mid K_{1}\right)+\mathcal{L}_{l}^{S}\left(\phi,\left|\mathcal{S}_{j}\right|\right)\end{array}: j \in[2]\right) \in \mathscr{H} \mathscr{K}\left(p_{V_{1} W_{1} X_{1}} p_{V_{2} W_{2} X_{2}}\right)$,

$$
\mathcal{L}_{j}^{C}(\phi,|\mathcal{U}|)=h_{b}(\phi)+5 \phi \log |\underline{\mathcal{U N} \mathcal{W}}|+\left.|| \underline{\mathcal{U} \mathcal{W}}\right|^{3} \phi \log \frac{1}{\phi} .
$$

where, $\phi, \mathcal{L}_{l}^{S}\left(\phi,\left|\mathcal{S}_{j}\right|\right)$ are as defined in Thm 2 .

Remark 6: For simplicity and compact description, we derive a uniform upper bound on all the mutual-information quantities involved in the description of the Han-Kobayashi region. This explains the large constant multiplying $\phi \log \frac{1}{\phi}$. Our third step is to use the decoded fixed B-L channel codewords towards conditional decoding of the outer code. The outer code is built on $\mathcal{X}_{j}$ and is superimposed on (interleaved vectors of) $C_{U}$. The challenge is that a fraction $\phi$ of the decoded codewords are erroneous. The approach is to treat the interleaved columns of the decoded $\hat{U}$ as a noisy state/side information. Interleaving ensures that these sub-vectors have a S-L IID pmf. Proof is similar to [12, Proof of Thm. 1].

Theorem 4: $\left(\underline{\mathcal{S}}, \mathbb{W}_{\underline{S}}\right)$ is transmissible over an IC $\mathbb{W}_{\underline{Y} \mid \underline{X}}$ if there exists (i) a finite set $\mathcal{K}$, maps $f_{j}: \mathcal{S}_{j} \rightarrow \mathcal{K}$, with $K_{j}=$ $f_{j}\left(S_{j}\right)$ for $j \in[2]$, (ii) $l \in \mathbb{N}, \delta>0$, (iii) finite set $\mathcal{U}$ and pmf $p_{U} p_{X_{1} \mid U} p_{X_{2} \mid U}$ defined on $\mathcal{U} \times \underline{\mathcal{X}}$, where $p_{U}$ is a type of sequences in $\mathcal{U}^{l}$, (iv) $A, B \geq 0, \rho \in(0, A)$ such that $\phi \in$ $[0,0.5)$, where

$$
\begin{array}{r}
A+B \geq(1+\delta) H\left(K_{1}\right), \text { and for } j \in[2], \\
B+H\left(S_{j} \mid K_{1}\right)+\mathcal{L}^{S}(\phi,|\mathcal{K}|)<I\left(X_{j} ; Y_{j} \mid U\right)-\mathcal{L}_{j}^{C}(\phi,|\mathcal{U}|) \\
\mathcal{L}_{j}^{C}(\phi,|\mathcal{U}|)=h_{b}(\phi)+\phi \log |\mathcal{U}|+\left|\mathcal{X}_{j}\right||\mathcal{Y}||\mathcal{U}|\left(1+\left|\mathcal{X}_{\dot{j}}\right|\right) \phi \log \frac{1}{\phi}
\end{array}
$$

and $\phi, \mathcal{L}^{S}(\phi,|\mathcal{K}|)$ are as defined in Thm 2

The final step in our generalization will combine the techniques of Thm. 3, 4. In particular, we employ Han-Kobayashi technique in the superposition layer. The message to be communicated through the outer code is split into private and public parts and coded using separate codebooks. Decoder $j$ uses the decoded fixed B-L channel codeword and employs a conditional Han-Kobayashi decoder. We omit a characterization in the interest of brevity.

\section{REFERENCES}

[1] W. Liu and B. Chen, "Interference Channels with Arbitrarily Correlated Sources," Trans. on Info. Th., vol. 57, no. 12, pp. 8027-8037, Dec 2011.

[2] G. Dueck, "A note on the multiple access channel with correlated sources (corresp.)," Trans. on Info. Th., vol. 27, no. 2, pp. 232 - 235, mar 1981.

[3] T. Cover, A. Gamal, and M. Salehi, "Multiple access channels with arbitrarily correlated sources," Trans. on Info. Th., pp. $648-657$, Nov 1980.

[4] K. Khezeli and J. Chen, "A Source-channel Separation theorem with application to the source broadcast problem," IEEE Trans. Info. Th., vol. 62, no. 4, pp. 1764-1781, 2016.

[5] Y. Murin, R. Dabora, and D. Gunduz, "On Joint Source-Channel Coding for Correlated sources over Multiple-Access Relay Channels," Trans. on Info. Th., vol. 60, no. 10, pp. 6231-6253, Oct 2014

[6] A. Lapidoth and M. Wigger, "A necessary condition for the transmissibility of correlated sources over a MAC," in 2016 ISIT, July 2016.

[7] P. Minero, S. H. Lim, and Y.-H. Kim, "A unified approach to Hybrid Coding," Trans. on Info. Th., vol. 61, no. 4, pp. 1509-1523, April 2015.

[8] A. Lapidoth and S. Tinguely, "Sending a Bivariate Gaussian over a Gaussian MAC," Trans. on Info. Th., vol. 56, pp. 2714-2752, 2010.
[9] C. Tian, S. Diggavi, and S. Shamai, "The Achievable Distortion Region of Sending a Bivariate Gaussian Source on the Gaussian Broadcast channel," Trans. on Info. Th., vol. 57, no. 10, pp. 6419-6427, Oct 2011.

[10] I. E. Aguerri and D. Gündüz, "Correlated Gaussian sources over Gaussian weak interference channels," in 2015 IEEE ITW, Oct 2015, pp. $84-88$.

[11] A. Padakandla, "Communicating correlated sources over a MAC in the absence of a Gács-Körner common part," in 2016 IEEE International Symposium on Information Theory (ISIT), July 2016, pp. 2014-2018.

[12] - "Communicating Correlated Sources Over a MAC in the absence of a Gács-Körner Common Part," available at http://arxiv.org/abs/1601.07091 .

[13] _ "Communicating Correlated Sources Over an Interference Channel," available at https://arxiv.org/abs/1701.06977 .

[14] T. Cover, A. Gamal, and M. Salehi, "Multiple access channels with arbitrarily correlated sources," Information Theory, IEEE Transactions on, vol. 26, no. 6 , pp. $648-657$, nov 1980.

[15] I. Csiszár and J. Körner, Information Theory: Coding Theorems for Discrete Memoryless Systems, 2nd ed. Cambridge Univ. Press, 2011.

[16] F. Shirani and S. Pradhan, "Finite block-length gains in distributed source coding," in Proc. IEEE 2014 ISIT, June 2014, pp. 1702-1706.

[17] F. Chaharsooghi, A. Sahebi, and S. Pradhan, "Distributed source coding in absence of common components," in Proc. 2013 ISIT, pp. 1362-66. 